\title{
The Arcetri Spectral Code for optically thin plasmas
}

\author{
E. Landi ${ }^{1}$ and M. Landini ${ }^{2}$ \\ 1 E.O. Hulburt Center for Space Research, NRL, Washington DC 20375, USA \\ 2 Dipartimento di Astronomia e Scienza dello Spazio, Universita' di Firenze, Firenze, Italy \\ Received 20 November 2001 / Accepted 8 January 2002
}

\begin{abstract}
The Arcetri Spectral Code allows one to evaluate the spectrum of the radiation emitted by hot and optically thin plasmas in the spectral range 1-2000 $\AA$. The Arcetri Code consists of a series of files that contain the emissivity of the plasma as a function of electron temperature and density. Both line and continuum emission are considered. These quantities are calculated using a database of atomic data and transition probabilities, mostly taken from the CHIANTI database. In the present work we describe the updates to the spectrum and present the new results. A comparison with the previous version of the code allows us to assess the improvements to the spectrum; comparison with other spectral codes allows us to assess the completeness of the Arcetri Code and of the CHIANTI database.
\end{abstract}

Key words. atomic data - plasmas - Sun: atmosphere - stars: atmospheres - ultraviolet: general astronomical data bases: miscellaneous

\section{Introduction}

The Arcetri Spectral Code was first created by M. Landini and B. C. Monsignori Fossi in the early 1970s, with the aim of developing a numerical code to evaluate the emissivity of a hot, optically thin plasma. The calculation was carried out under the coronal approximation for a number of lines in the 1-100 $\AA$ spectral range. The coronal approximation calculates the upper level population by assuming collisional and radiative coupling of that level with the ground level only: in several cases it is a reliable approximation, but for complex ions it may lead to significant inaccuracies. The first updated version of the spectrum also included the continuum radiation from a few important ions (Landini \& Monsignori Fossi 1990). This version of the spectrum has been extensively used by the authors to study the solar X-ray emission measured by the SOLRAD satellites equipped with broad band detectors and for predictions of X-ray and EUV emission from coronae of solar type stars.

Since then, the Arcetri Spectral Code has undergone a continuous updating process, in order to expand the atomic database, include more lines in the X-ray wavelength range, update the atomic parameters in the Code and extend the wavelength range covered by the spectrum.

Landini \& Monsignori Fossi (1990) published a version of the Code that calculated line and continuum emission in the 1-2000 $\AA$ wavelength range, included the twophoton, free-free and free-bound continuum from the most

Send offprint requests to: E. Landi,

e-mail: landi@poppeo.nrl.navy.mil astrophysically important ions, and developed a suite of programs that allowed them to determine the Differential Emission Measure (DEM) of the emitting plasma from the observations. This version of the Code has been extensively applied to the analysis of X-ray and EUV spectra of the solar corona, and to investigate the $\mathrm{X}$ and EUV emission of solar-type stars. A version of this Code covering the 70-700 $\AA$ spectral range has been used for data reduction of observations of the Extreme Ultraviolet Explorer (EUVE) satellite.

The increased quantity of atomic data and transition rates available in the literature has led Monsignori Fossi \& Landini (1994a,b) to abandon the coronal approximation in order to calculate level population and line emissivities in a more accurate way. A database has been created including energy levels, wavelengths, radiative transition probabilities and excitation rates that allowed the calculation of level populations by assuming statistical balance between collisional excitation and de-excitation and radiative decay between all the levels of each ion. Monsignori Fossi \& Landini (1994a,b) included data for the iron ions only. Subsequently this approach was extended to all the other ions, and the coronal approximation was retained only for the transitions coming from levels for which the data necessary for the statistical balance calculations were not available. Most of this work was carried out as a collaboration with the Scientific Team of the Coronal Diagnostic Spectrometer (Harrison et al. 1995) on SOHO, as described in Monsignori Fossi \& Landini (1994c).

The data developed so far for the Arcetri Code have been completely re-assessed, extended and updated in 
order to be inserted into the CHIANTI database (Dere et al. 1997), which has represented a natural evolution of the Arcetri Code. CHIANTI is described elsewhere (Dere et al. 1997, 2001; Landi et al. 1999); here it is sufficient to say that CHIANTI consists of a database of atomic parameters, radiative coefficients and excitation rates that allows to solve the statistical equilibrium equations for level populations for most of the astrophysically abundant ions.

A complete update of the Arcetri Code, including the CHIANTI version 1.01 data for wavelengths greater than $50 \AA$ and all the data for the minor ions has been completed by Landi \& Landini (1998) and released on the internet. In that version of the Code, X-ray lines were still calculated in the coronal approximation.

The CHIANTI project has been continually developed and maintained, and now it has been extended to wavelengths shorter than $50 \AA$ by including data for the helium and hydrogen isoelectronic sequences as well as data for transitions from high excitation configurations and satellite lines (Dere et al. 2001).

The present update of the Code has been made in order to include the CHIANTI data for X-ray transitions, that replace and improve most of the existing data from the older version of the Code. CHIANTI version 3.03 is used. CHIANTI emissivities, calculated by solving the equations of detailed balance for level population of each ion, are considered to be much more accurate than those obtained through the coronal approximation, and allows the evaluation of density dependent level populations. Also, CHIANTI version 3.03 data include improved data for several individual ions.

What distinguishes the Arcetri Code from CHIANTI is the fact that a lot of transitions not present in the CHIANTI database are calculated by the Arcetri Code using the coronal approximation. In the present version, there are some 1050 transitions calculated with this method at all wavelengths. For them, no data are available in the literature to solve the statistical balance equations. Most of them come from high-energy configurations in iron ions (X-ray lines) or from transitions involving $n=3$ and $n=4$ levels in several other ions.

The Spectral Code theoretical background and its atomic database are briefly described in Sect. 2. Results are presented in Sect. 3; Sect. 4 reports the comparison between the Code predicted intensities and the predictions of MEKAL code (Sect. 4.1), and the earlier version of the Code (Sect. 4.2). Conclusions are drawn in Sect. 5 .

\section{The line intensity}

The number of photons emitted in a spectral line $(i \rightarrow j)$ for an optically thin coronal plasma is given by:

$$
\begin{aligned}
I_{i j} & =\int_{V} N_{j}\left(X^{m}\right) A_{j i} \mathrm{~d} V \\
& =\int_{V} G_{i j}\left(T, N_{\mathrm{e}}\right) N_{\mathrm{e}}^{2} \mathrm{~d} V \quad \mathrm{ph} \mathrm{cm}^{-3} \mathrm{~s}^{-1}
\end{aligned}
$$

where $G_{i j}\left(T, N_{\mathrm{e}}\right)$ is the Contribution Function of the line and depends on the electron temperature $(T)$ mainly through the ion abundance, and on the electron density $\left(N_{\mathrm{e}}\right)$, mainly through the level population. The function $G_{i j}\left(T, N_{\mathrm{e}}\right)$ may be expressed as

$G\left(T, \lambda_{i, j}\right)=\frac{N_{j}\left(X^{m}\right)}{N\left(X^{+m}\right)} \frac{N\left(X^{m}\right)}{N(X)} \frac{N(X)}{N(H)} \frac{N(H)}{N_{\mathrm{e}}} \frac{A_{j i}}{N_{\mathrm{e}}}$

where

$-\frac{N_{j}\left(X^{+m}\right)}{N\left(X^{+m}\right)}$ is the relative upper level population;

$-\frac{N\left(X^{+m}\right)}{N(X)}$ is the relative abundance of the ion $X^{+m}$ (ion fraction);

$-\frac{N(X)}{N(H)}$ is the abundance of the element $X$ relative to hydrogen;

$-\frac{N(H)}{N_{\mathrm{e}}}$ is the hydrogen abundance relative to the electron density $(\approx 0.8)$.

Element abundances are known from the literature from a variety of different sources, such as Allen (1973), Feldman (1992), Meyer (1985), Grevesse \& Anders (1992), Waljeski et al. (1994); see Feldman \& Laming (2000) for more details. Ion fractions have been calculated by a number of authors, such as Shull \& Van Steenberg (1982), Arnaud \& Rothenflug (1985), Landini \& Monsignori Fossi (1991), Arnaud \& Raymond (1992) and Mazzotta et al. (1998).

Level populations must be calculated solving the statistical equilibrium equation including all the important processes involved in level excitation and de-excitation. In low density plasmas the most important populating and de-populating processes are spontaneous radiative decay, and excitation and de-excitation from electron-ion collisions, since they are generally faster than ionisations and recombinations. In hot plasmas proton collision rates, neglected in the present version of the Code, can also be important in determining the level population. The statistical equilibrium equations take the form:

$$
\begin{gathered}
N_{j}\left(N_{\mathrm{e}} \Sigma_{i} C_{j, i}^{\mathrm{e}}+N_{\mathrm{p}} \Sigma_{i} C_{j, i}^{\mathrm{p}}+\Sigma_{i<j} A_{j, i}\right)= \\
\Sigma_{i} N_{i}\left(N_{\mathrm{e}} C_{i, j}^{\mathrm{e}}+N_{\mathrm{p}} C_{i, j}^{\mathrm{p}}\right)+\Sigma_{i>j} N_{i} A_{i, j}
\end{gathered}
$$

with $C_{j, i}^{\mathrm{e}}$ and $C_{j, i}^{\mathrm{p}}$ the electron and proton collisional excitation rates $\left(\mathrm{cm}^{3} \mathrm{~s}^{-1}\right), C_{i, j}^{\mathrm{e}}$ and $C_{i, j}^{\mathrm{p}}$ the electron and proton collisional de-excitation rates and $A_{j i}\left(\mathrm{~s}^{-1}\right)$ are radiative decay probabilities from level $j$ to level $i$.

The collisional excitation rate for a Maxwellian electron velocity distribution can be expressed as

$C_{i, j}^{\mathrm{e}}=\frac{8.63 \times 10^{-6}}{T_{\mathrm{e}}^{1 / 2}} \frac{\Upsilon_{i, j}\left(T_{\mathrm{e}}\right)}{\omega_{i}} \exp \left(-\frac{\Delta E_{i, j}}{k T_{\mathrm{e}}}\right)$

where $\omega_{i}$ is the statistical weight of level $i, k$ is the Boltzmann constant and $\Upsilon_{i, j}$ is the thermally-averaged collision strength (effective collision strength):

$\Upsilon_{i, j}=\int_{0}^{\infty} \Omega_{i, j} \exp \left(-\frac{E}{k T_{\mathrm{e}}}\right) \mathrm{d} \frac{E}{k T_{\mathrm{e}}}$ 
where $\Omega_{i, j}$ is the collision strength, related to the electron excitation cross section and $E$ is the energy of the scattered electron relative to the final energy state of the ion.

For solving the statistical equilibrium in Eq. (3) it is necessary to have a large dataset which includes an atomic model with experimental energy levels and radiative and collisional transition probabilities for all the possible transitions within the levels of the adopted atomic model. For this reason the CHIANTI database has been developed. CHIANTI allows the complete solution of the system of equations described above for the most important ions. In the present update we have included the whole CHIANTI dataset (version 3.03) in the Arcetri Spectral Code, and we have also added original data for NeIII and NixxI, described below.

Also continuum emission from free-free, free-bound and two-photon processes can be calculated by the Arcetri Spectral Code. Since no changes have been done on the methods and approximations adopted for the calculation of the continuum from the previous version of the Code, the reader is referred to Landi \& Landini (1998) for further details.

\subsection{Ne III data}

The NeIII atomic model adopted in the present version of the Code includes the $2 \mathrm{~s}^{2} 2 \mathrm{p}^{4}, 2 \mathrm{~s} 2 \mathrm{p}^{5}, 2 \mathrm{p}^{6}, 2 \mathrm{~s}^{2} 2 \mathrm{p}^{3} 3 \mathrm{~s}$ and $2 \mathrm{~s}^{2} 2 \mathrm{p}^{3} 3 \mathrm{~d}$ configurations, for a total of 58 fine-structure levels. Experimental energies for most of the levels are taken from the NIST database (Fuhr et al. 1999); no value is available in the literature for the other levels in the model.

Theoretical energy levels and radiative data come from Bhatia et al. (2001a), and have been calculated using the SUPERSTRUCTURE computer program (Eissner et al. 1974). The A values for the lowest 5 levels of the atomic model are taken from Galavis et al. (1997), and the radiative data for the ${ }^{3} \mathrm{P}_{1}{ }^{-1} \mathrm{~S}_{0}$ and ${ }^{1} \mathrm{D}_{2}{ }^{-1} \mathrm{~S}_{0}$ in the ground configuration come from the laboratory measurements of Daw et al. (2000).

Collisional data for the ground configuration come from the R-Matrix calculation of Butler \& Zeippen (1994), carried out as part of the Iron Project. Maxwellianaveraged collision strengths are provided for temperatures in the $10^{3}-10^{4} \mathrm{~K}$; this dataset is described in Dere et al. (1997). Excitation rates for transitions involving all the other levels have been taken from Bhatia et al. (2001a). Collision strengths are available for 5 values of the incident electron energy between 5 and 45 Ry. The Bhatia et al. (2001a) dataset is the only one that includes $n=3$ configurations, and has been calculated using the Distorted Wave approximation (Eissner \& Seaton 1972). Data for the ground configuration were also available, but in this case resonant contributions to the collision strengths are important, so that the R-Matrix data from Butler \& Zeippen (1994) are believed to be more accurate.

\subsection{Ni XXI data}

Bhatia et al. (2001b) provide the only Nixxi complete dataset available in the literature, despite lines from this ion have long been observed in the past. The atomic model adopted for this ion includes the same configurations and levels in the Ne III dataset. Experimental energy levels come from the recent compilation of Shirai et al. (2000), while theoretical values are provided by Bhatia et al. (2001b).

Radiative and collisional data come from Bhatia et al. (2001b). A values and oscillator strengths have been calculated using the SUPERSTRUCTURE computer program. Collision strengths are computed adopting the Distorted Wave approximation for 5 values of the incident electron energy between 85 and $425 \mathrm{Ry}$.

\section{Results}

The Arcetri Code allows to evaluate the level population and Contribution Functions for any temperature ranging between $10^{4}$ and $10^{8} \mathrm{~K}$ and any electron density.

In order to calculate the Contribution Function curves, we have adopted electron density values between $\log N_{\mathrm{e}}=$ 2 to $\log N_{\mathrm{e}}=14$, in steps of $\log N_{\mathrm{e}}=1\left(N_{\mathrm{e}}\right.$ in $\left.\mathrm{cm}^{-3}\right)$; such values allow the tables provided at the address below to be used for studies of most astrophysical objects. Data are provided in the $1-2000 \AA$ wavelength range, and for temperatures between $1 \times 10^{4} \mathrm{~K}$ and $1 \times 10^{8} \mathrm{~K}$. The ion fractions of Mazzotta et al. (1998) and the coronal abundances of Feldman (1992) have been used in the calculation.

Tables with the results are freely available for general use at the following Internet address:

\section{http://www.arcetri.astro.it/science/spettro/ spettro.html}

For each electron density value, separate tables are provided that include:

1. Contribution Function for each line in the database;

2. line emissivities integrated over steps of $0.5 \AA$;

3. continuum emissivities integrated over steps of $0.5 \AA$;

4. total plasma emissivity integrated over steps of $0.5 \AA$;

5. radiative losses for the most important elements.

The results are provided as a function of the electron temperature. Radiative losses are provided for $N_{\mathrm{e}}=$ $10^{10} \mathrm{~cm}^{-3}$ only, since the effects of density are within $10 \%$. All data are provided per unit emission measure, both in erg $\mathrm{cm}^{3} \mathrm{~s}^{-1}$ and phot $\mathrm{cm}^{3} \mathrm{~s}^{-1}$; emissivity per unit volume is obtained by multiplying the results by $N_{\mathrm{e}}^{2}$.

An example of the emission as calculated from the new version of the Code can be found in Figs. 1 to 4.

\subsection{Range of validity of the Arcetri Code}

Like the other available codes, the Arcetri Code relies on several assumptions that in some cases limit the accuracy 


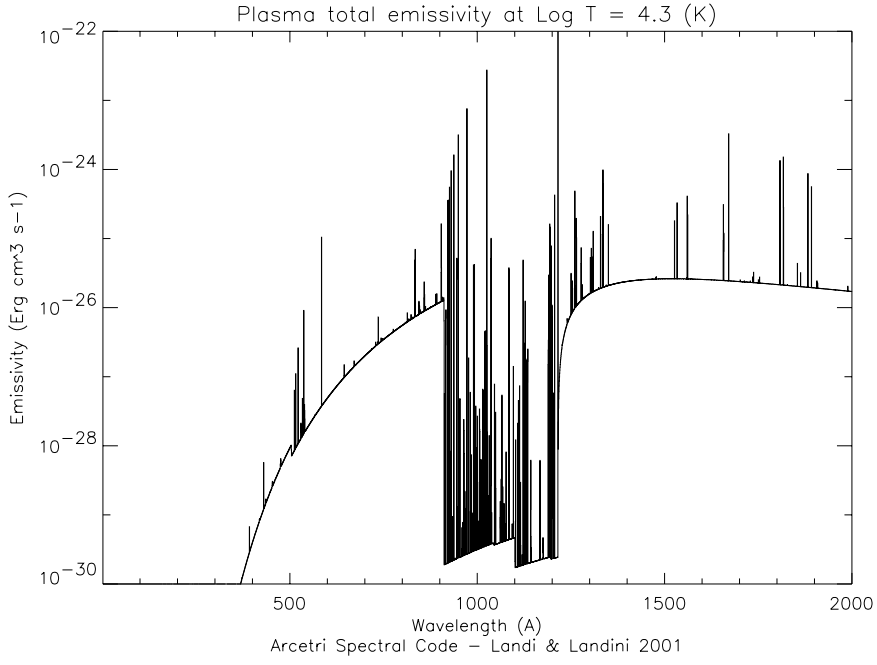

Fig. 1. Spectral emissivity integrated over spectral bins $0.5 \AA$ wide. The electron temperature is $\log T=4.3(T$ in $\mathrm{K})$; the electron density is $\log N_{\mathrm{e}}=2\left(N_{\mathrm{e}}\right.$ is $\left.\mathrm{cm}^{-3}\right)$.

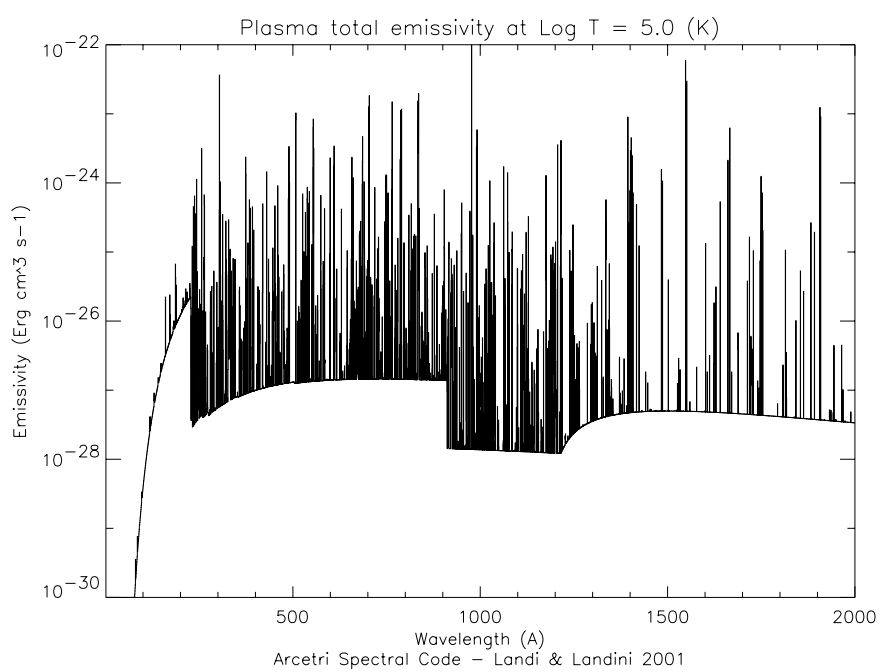

Fig. 2. Spectral emissivity integrated over spectral bins $0.5 \AA$ wide. The electron temperature is $\log T=5.0(\mathrm{~T}$ in $\mathrm{K})$; the electron density is $\log N_{\mathrm{e}}=4\left(N_{\mathrm{e}}\right.$ is $\left.\mathrm{cm}^{-3}\right)$.

of its predictions and the range of its validity. Here we briefly discuss them.

The plasma has been assumed to be optically thin. However, at high densities opacity effects may arise. For example, Brooks et al. (2000) found that in the lower transition region the line of a few ions are affected by opacity; other situations occur in solar plasmas as well as in other astrophysical sources. The upper limit chosen for electron density $\left(N_{\mathrm{e}} \leq 10^{14} \mathrm{~cm}^{-3}\right)$ is low enough to avoid selfabsorption of resonance lines. For a few ions, photoexcitation in transitions within the ground configurations may influence level populations in the presence of strong radiation fields.

At temperatures lower than $10^{5} \mathrm{~K}$ helium and hydrogen begin to recombine, so that the $\frac{N(\mathrm{H})}{N_{\mathrm{e}}}$ ratio departs from the adopted value of 0.8 , typical of fully ionized

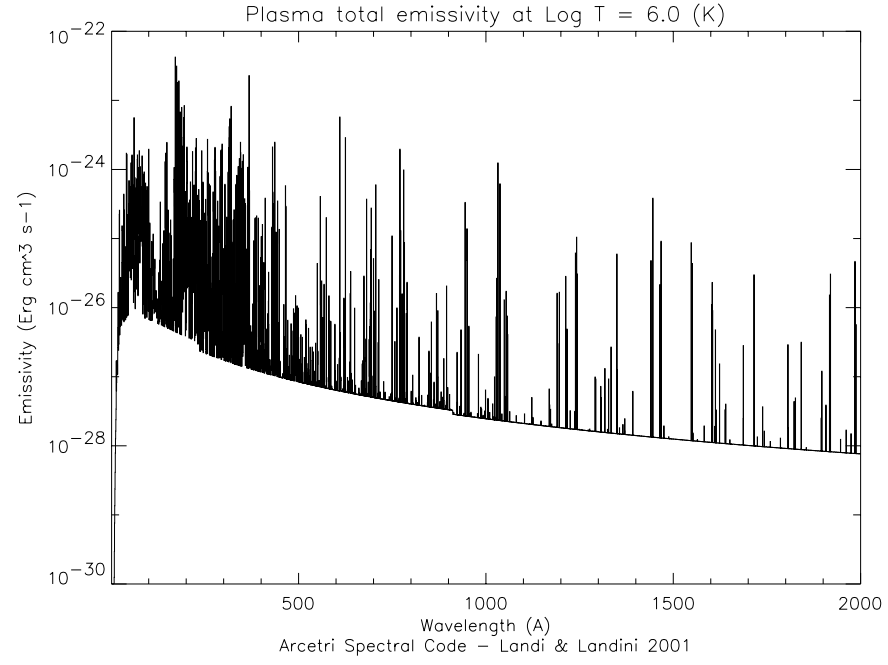

Fig. 3. Spectral emissivity integrated over spectral bins $0.5 \AA$ wide. The electron temperature is $\log T=6.0(T$ in $\mathrm{K})$; the electron density is $\log N_{\mathrm{e}}=8\left(N_{\mathrm{e}}\right.$ is $\left.\mathrm{cm}^{-3}\right)$.

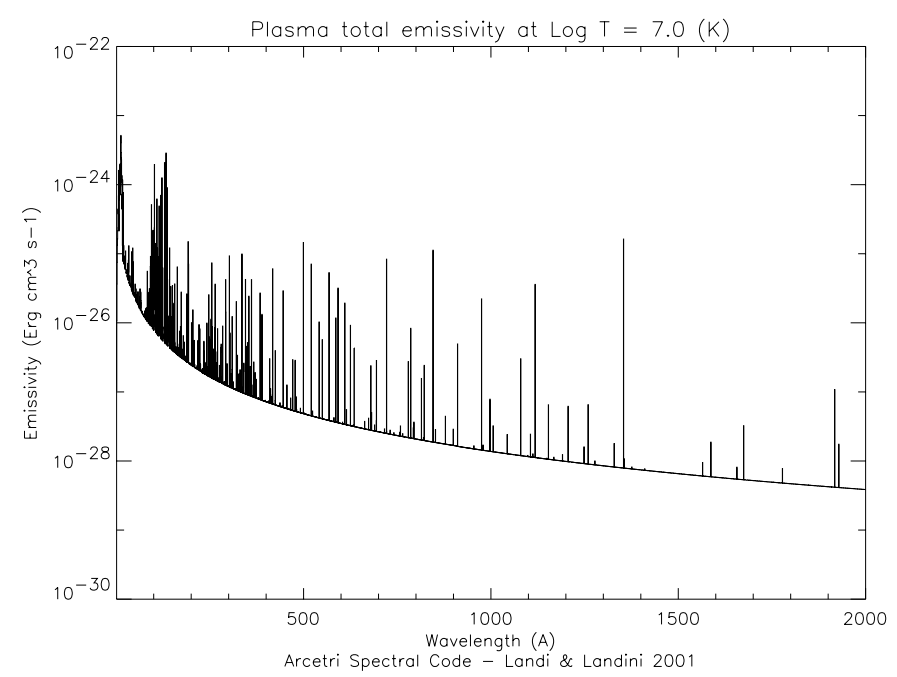

Fig. 4. Spectral emissivity integrated over spectral bins $0.5 \AA$ wide. The electron temperature is $\log T=7.0$ ( $T$ in $\mathrm{K})$; the electron density is $\log N_{\mathrm{e}}=11\left(N_{\mathrm{e}}\right.$ is $\left.\mathrm{cm}^{-3}\right)$.

plasmas. For this reason, line emissivities in that temperature range may be overestimated.

At temperatures greater than $10^{7} \mathrm{~K}$ proton rates effects, not accounted for in the Arcetri Code, are important in the calculation of level populations of some ions. The upper limit set to the electron temperature $\left(T<10^{8} \mathrm{~K}\right)$ allows us to neglect the relativistic corrections to the bremsstrahlung cross sections.

The assumption of ionization equilibrium is questionable in highly transient plasmas, since plasma evolution timescales may become comparable to ionization and recombination times, so that some caution is required when analysing spectra from rapidly evolving sources.

The ion fractions adopted in the present work are the most accurate available in the literature, and yet they do not take into account the effects of electron density in dielectronic recombination, and they are provided as a 
function of electron temperature only. However, Reisenfield et al. (1992) have shown that electron density effects may alter significantly the ion fractions of Li-like ions, and argue that similar effects can be expected for Na-like line also. Neglecting these effects leads to inaccurate theoretical emissivities for these ions; indeed, disagreement with observations has been found by several authors (Landi et al. 2002; Del Zanna 1999; Dupree 1972). Recombination to excited levels, not accounted for in the present version of the Code, also may affect the He-like line ratios in the $\mathrm{X}$-ray wavelength range, as shown by Porquet et al. (2001).

On the overall, the Arcetri Code is most accurate in the $10^{5}-10^{7} \mathrm{~K}$ temperature range, and provides accurate estimates of plasma emissivities for most ions up to $10^{8} \mathrm{~K}$.

\section{Comparison with other codes}

In the last few years, several groups have developed atomic databases that allow calculation of plasma emissivities, to be used for general plasma diagnostic purposes or to be included as part of the analysis software of some instrument. The main codes available in the literature are

- MEKAL (Mewe et al. 1995);

- APEC/APED (Smith et al. 2001);

- XSTAR (Bautista \& Kallman 2001);

- PINTofALE (Drake \& Kashyap 2000).

All these, with the exception of the MEKAL Code, make extensive use of the CHIANTI database, sometimes complementing CHIANTI data with other literature sources. The XSTAR database includes also ionization and recombination data that allow the user to take into account ionization and recombination in level population. APEC/APED complements CHIANTI data with unpublished Distorted Wave calculations obtained with the HULLAC Code (Bar-Shalom et al. 2001). Of all these, the MEKAL Code is the only one that uses completely independent data from the Arcetri Code and CHIANTI, and thus it is the most useful to compare with the present results.

We have also compared the present version of the Code with results from the previous version, so that it is possible to assess the differences given by the improvements in the emission line dataset.

Figures 5 to 11 display a sample comparison between the present version of the Code, the old version and the MEKAL code, for a few spectral ranges and for a few sample temperatures. The Arcetri Code calculations have been made assuming $\log N_{\mathrm{e}}=10.0\left(N_{\mathrm{e}}\right.$ in $\left.\mathrm{cm}^{-3}\right)$; all three spectra have been calculated by using the element abundances reported by Grevesse \& Sauval (1998), the ion fractions Arnaud \& Raymond (1992) for Fe, and those of Arnaud \& Rothenflug (1985) for the other elements.

\subsection{Comparison with the MEKAL spectrum}

The comparison between the Arcetri Code and the MEKAL Code (version 1.1) reveals some significant
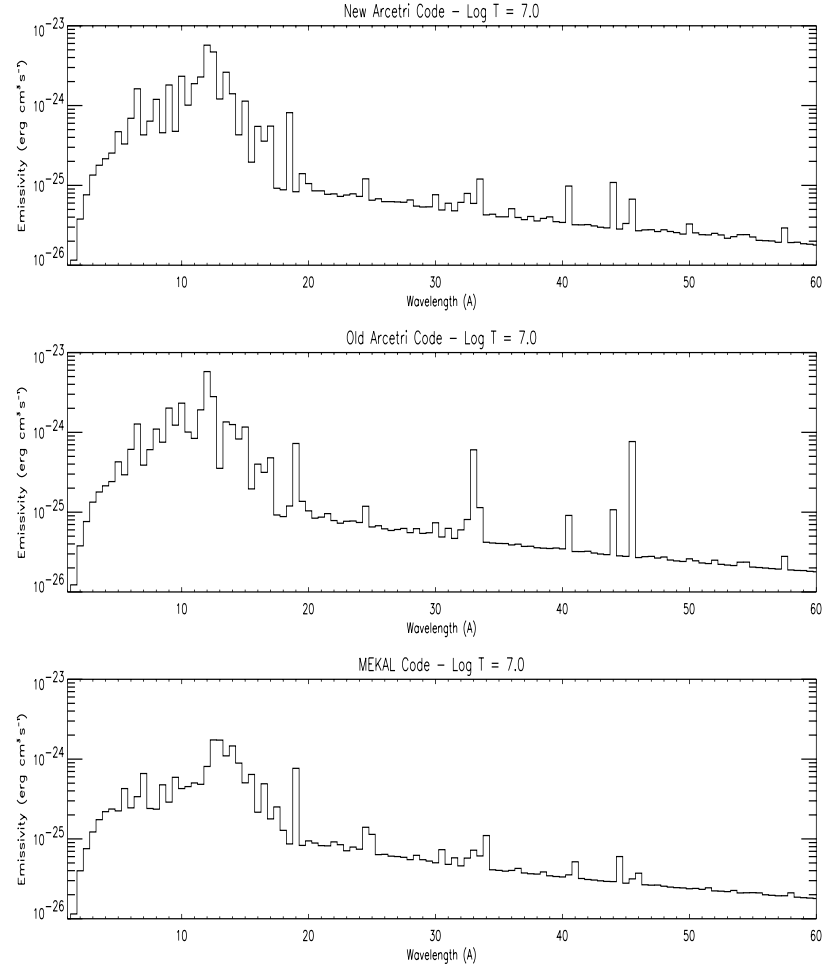

Fig. 5. Comparison of emissivities evaluated by the new version of the Arcetri Code (top), the old version of the Arcetri Code (middle) and the MEKAL spectrum (bottom), with $\log T=7.0(T$ in $\mathrm{K})$, and $\log N_{\mathrm{e}}=10\left(N_{\mathrm{e}}\right.$ in $\left.\mathrm{cm}^{-3}\right)$. The emissivity has been integrated over spectral bins each $0.5 \AA$ wide.
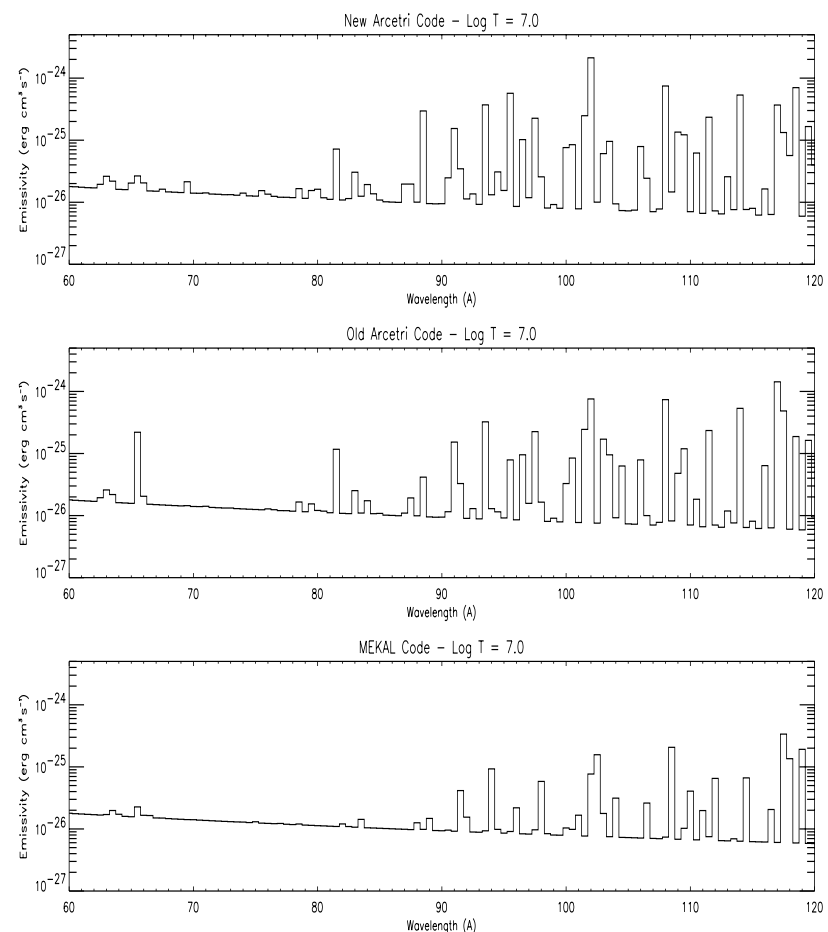

Fig. 6. Comparison of emissivities evaluated by the new version of the Arcetri Code (top), the old version of the Arcetri Code (middle) and the MEKAL spectrum (bottom), with $\log T=7.0(T$ in $\mathrm{K})$, and $\log N_{\mathrm{e}}=10\left(N_{\mathrm{e}}\right.$ in $\left.\mathrm{cm}^{-3}\right)$. The emissivity has been integrated over spectral bins each $0.5 \AA$ wide. 

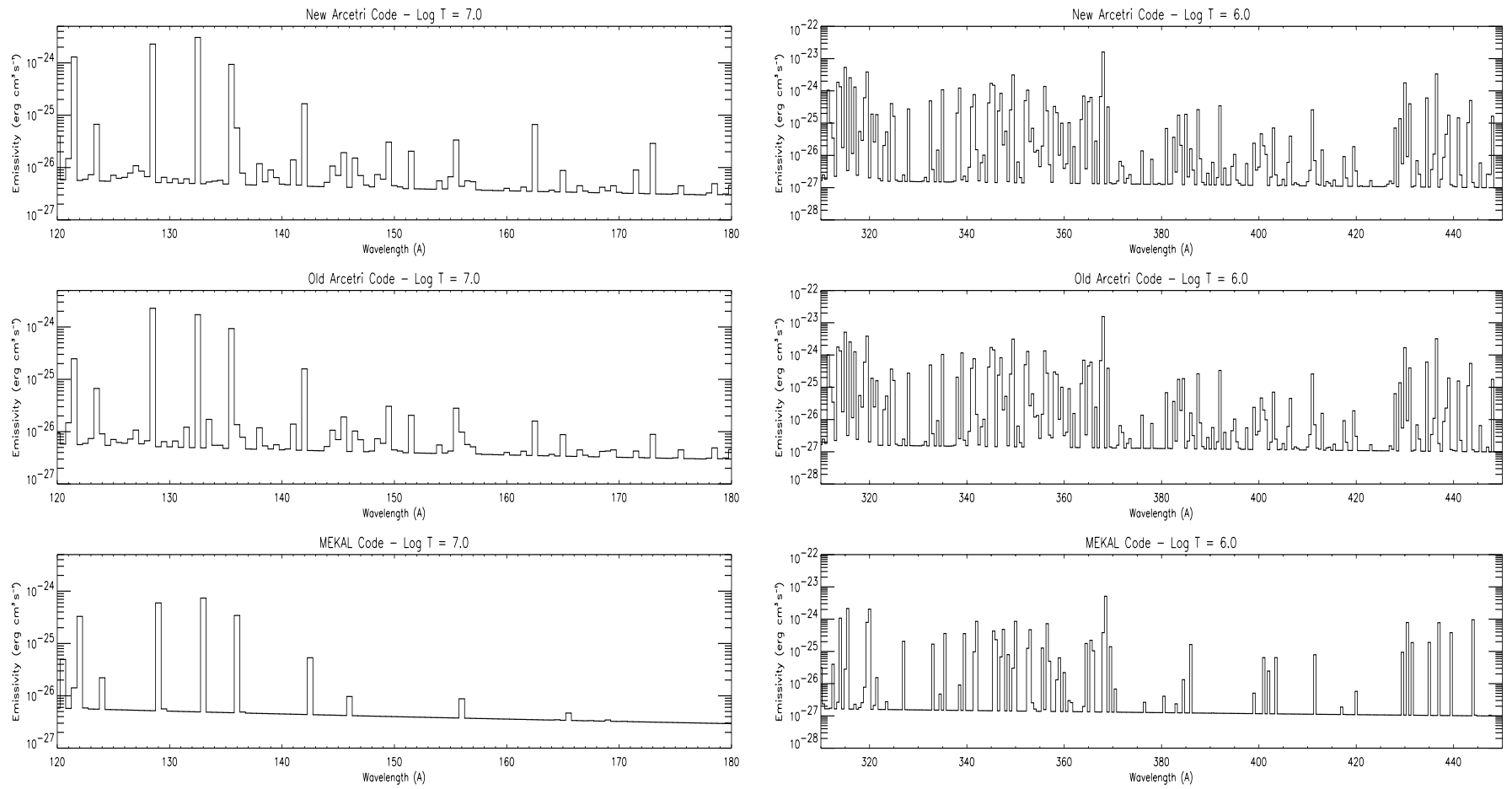

Fig. 7. Comparison of emissivities evaluated by the new version of the Arcetri Code (top), the old version of the Arcetri Code (middle) and the MEKAL spectrum (bottom), with $\log T=7.0(T$ in $\mathrm{K})$, and $\log N_{\mathrm{e}}=10\left(N_{\mathrm{e}}\right.$ in $\left.\mathrm{cm}^{-3}\right)$. The emissivity has been integrated over spectral bins each $0.5 \AA$ wide.
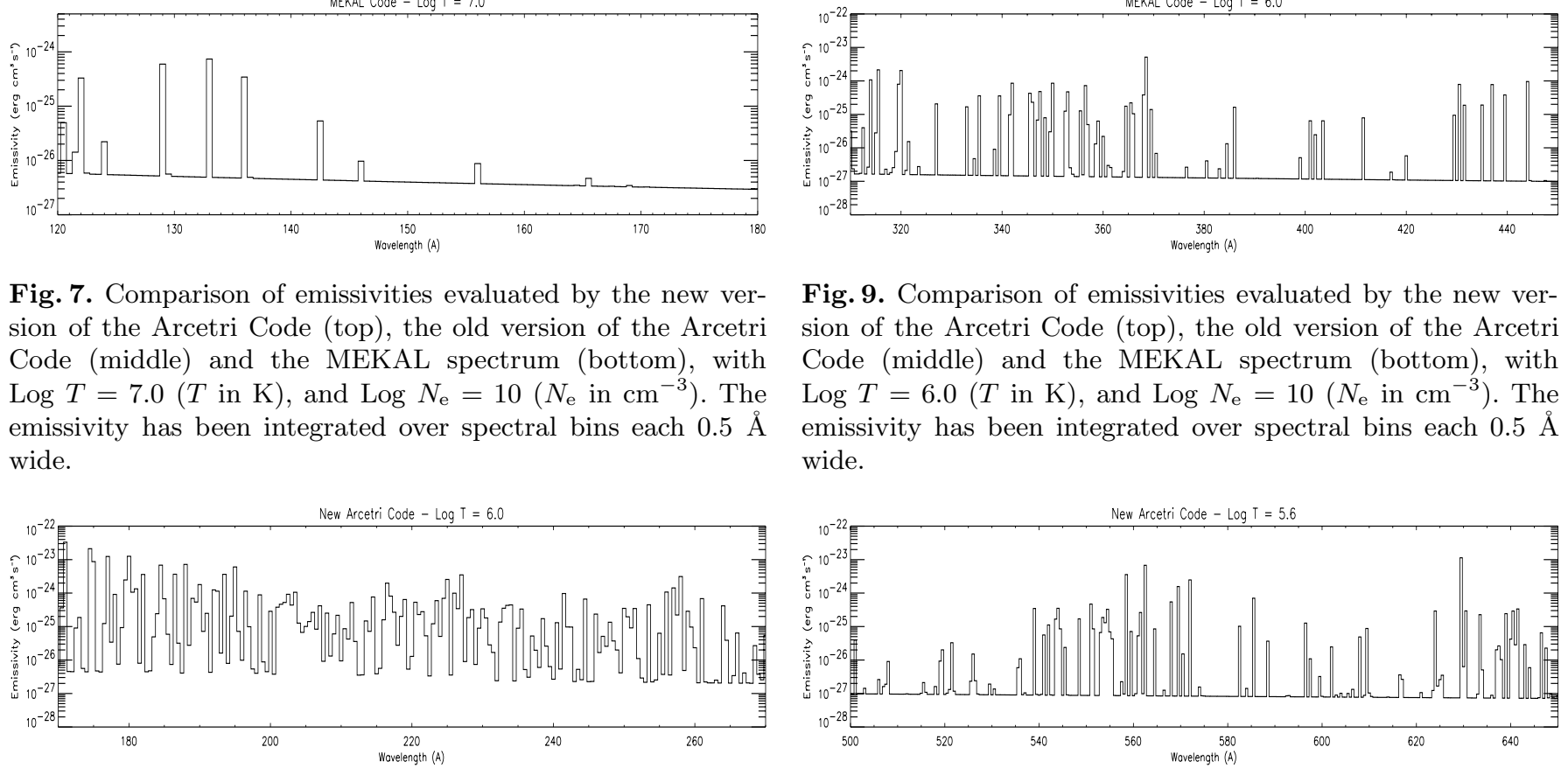

Fig. 9. Comparison of emissivities evaluated by the new version of the Arcetri Code (top), the old version of the Arcetri Code (middle) and the MEKAL spectrum (bottom), with $\log T=6.0(T$ in $\mathrm{K})$, and $\log N_{\mathrm{e}}=10\left(N_{\mathrm{e}}\right.$ in $\left.\mathrm{cm}^{-3}\right)$. The emissivity has been integrated over spectral bins each $0.5 \AA$ wide.
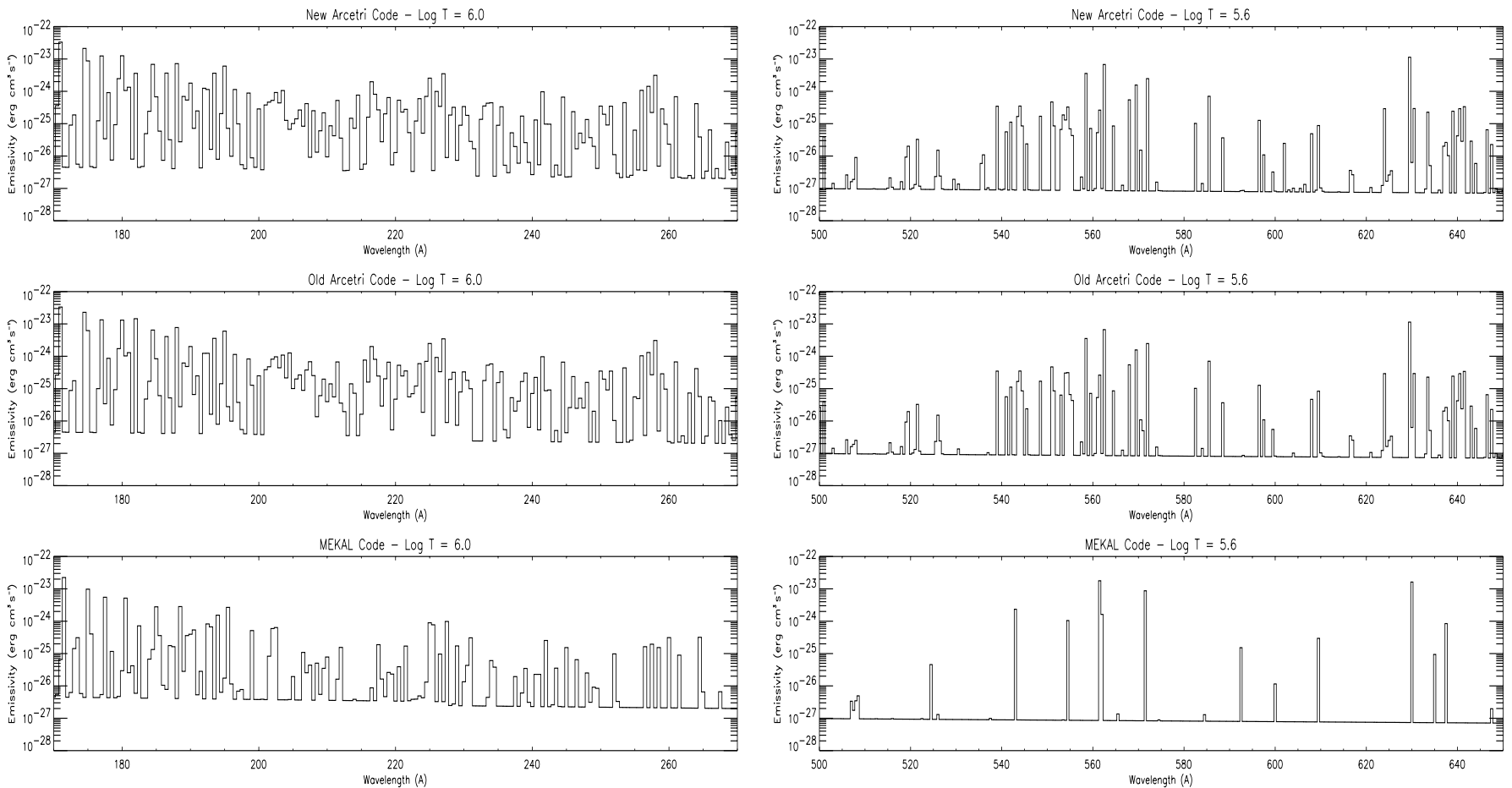

Fig. 8. Comparison of emissivities evaluated by the new version of the Arcetri Code (top), the old version of the Arcetri Code (middle) and the MEKAL spectrum (bottom), with $\log T=6.0(T$ in $\mathrm{K})$, and $\log N_{\mathrm{e}}=10\left(N_{\mathrm{e}}\right.$ in $\left.\mathrm{cm}^{-3}\right)$. The emissivity has been integrated over spectral bins each $0.5 \AA$ wide.

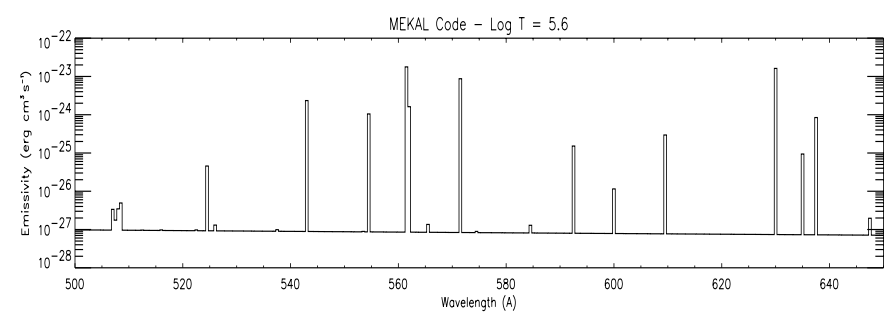

Fig. 10. Comparison of emissivities evaluated by the new version of the Arcetri Code (top), the old version of the Arcetri Code (middle) and the MEKAL spectrum (bottom), with $\log T=5.6(T$ in $\mathrm{K})$, and $\log N_{\mathrm{e}}=10\left(N_{\mathrm{e}}\right.$ in $\left.\mathrm{cm}^{-3}\right)$. The emissivity has been integrated over spectral bins each $0.5 \AA$ wide. 

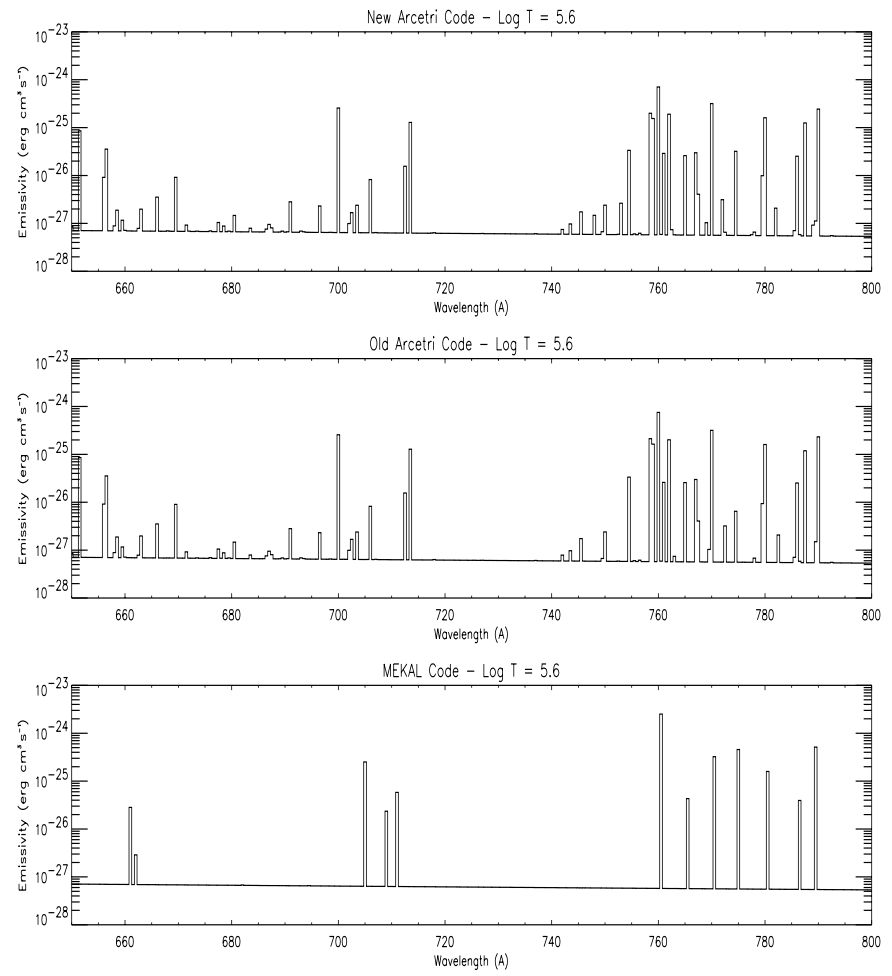

Fig. 11. Comparison of emissivities evaluated by the new version of the Arcetri Code (top), the old version of the Arcetri Code (middle) and the MEKAL spectrum (bottom), with $\log T=5.6(T$ in $\mathrm{K})$, and $\log N_{\mathrm{e}}=10\left(N_{\mathrm{e}}\right.$ in $\left.\mathrm{cm}^{-3}\right)$. The emissivity has been integrated over spectral bins each $0.5 \AA$ wide.

differences. On the overall, it appears from Figs. 5 to 11 that at wavelengths longer than $60 \AA$ the Arcetri Code predicts many more transitions than the MEKAL code. It is however to be pointed out that the latter code is able to account for nearly all the strongest features up to around $380 \AA$ A. For longer wavelengths, MEKAL accounts only for the very strongest spectral features, and misses nearly all the weaker lines that are predicted by CHIANTI. Many of these transitions have been observed in the solar spectrum.

At X-ray wavelengths the MEKAL code is rather complete, and it seems to be even more complete than the Arcetri Code. A detailed comparison between the two is displayed in Fig. 12, where the percentual difference between the two computations is also shown. The largest disagreement between the two computations is found between $10 \AA$ and $16 \AA$. The highest emissivity predicted by MEKAL between $13 \AA$ and $16 \AA$ is most probably due to the transitions coming from high-energy configurations $(n=4,5)$ for highly ionized iron, for which the MEKAL code database is more complete than the CHIANTI/Arcetri one; the MEKAL code incorporates unpublished calculations obtained with the HULLAC Code, that are unavailable in the literature.
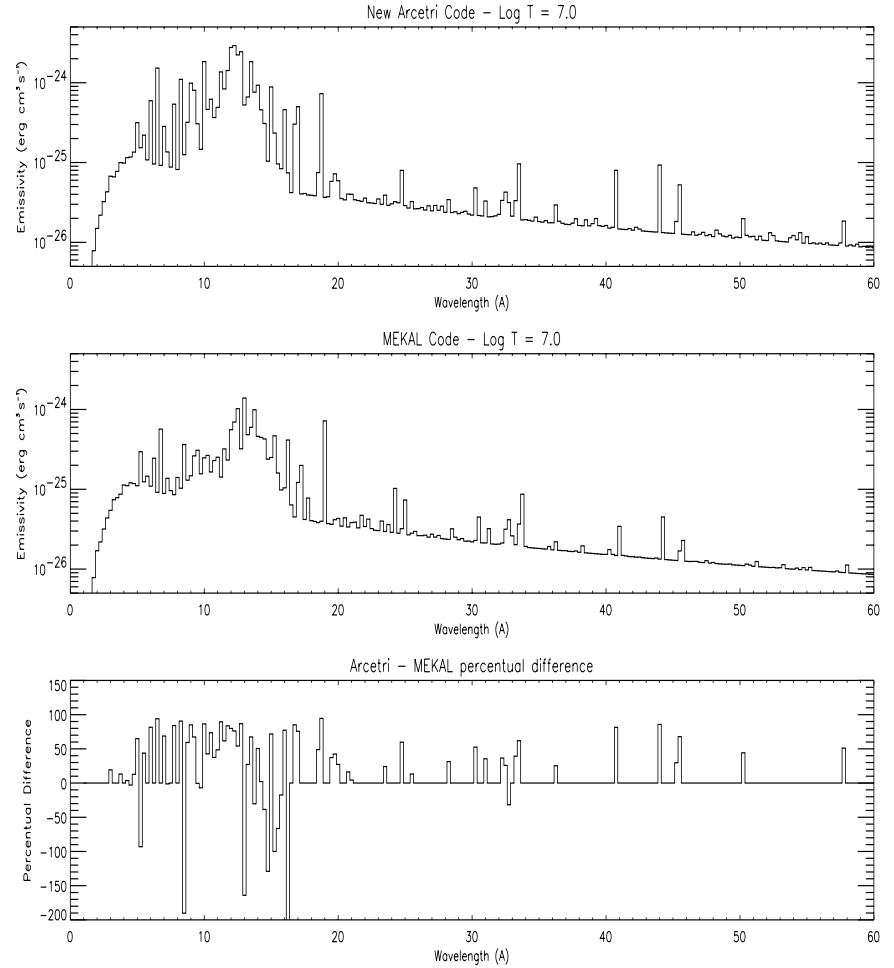

Fig. 12. Percentual difference between the new version of the Arcetri Code and the MEKAL code, calculated with $\log T=$ $7.0(T$ in $\mathrm{K})$, and $\log N_{\mathrm{e}}=10\left(N_{\mathrm{e}}\right.$ in $\left.\mathrm{cm}^{-3}\right)$. The emissivity has been integrated over spectral bins each $0.25 \AA$ wide.

\subsection{Comparison with the old Arcetri Code}

The differences between the two versions of the Code are displayed in Fig. 13, where the ratio of their emissivities is shown as a function of wavelength. In Fig. 13 the spectrum is divided in four segments, and for each of them the plasma temperature has been assumed to be the temperature where most of the spectral lines available in each range are emitted. This is $\log T=7.0$ in the $0-200 \AA$ range, $\log T=6.0$ in the $200-600 \AA$ range, $\log T=5.0$ in the $600-1300 \AA$ range and $\log T=4.5$ in the 1300 $2000 \AA$ range. Only the strongest lines (emissivity larger than $1 \times 10^{-27}$ erg $\mathrm{cm}^{3} \mathrm{~s}^{-1}$ ) have been considered.

The complete replacement of the old X-ray database with CHIANTI data has caused moderate discrepancies below $50 \AA$. The greater discrepancies are due to several transitions not included in the old code, as well as changes in the wavelengths of other transitions that were already included in the old version of the code. The revision of the dataset for the FexVIII-XXIV ions done in CHIANTI version 3 has led to more substantial differences in the $100 \AA$ region, where most of the allowed $n=2 \rightarrow n=$ 2 transitions from these ions are found.

Differencies arise in the $200-270 \AA$ region at one million degrees, and these are mostly due to the change in the atomic data for a few of the Fe ions (Fe X-XIV) whose strong lines emit in this wavelength range. Also several transitions from C-like, N-like and O-like ions for $\mathrm{Mg}$, Si and $\mathrm{S}$ are found in this range: the differences that some of 

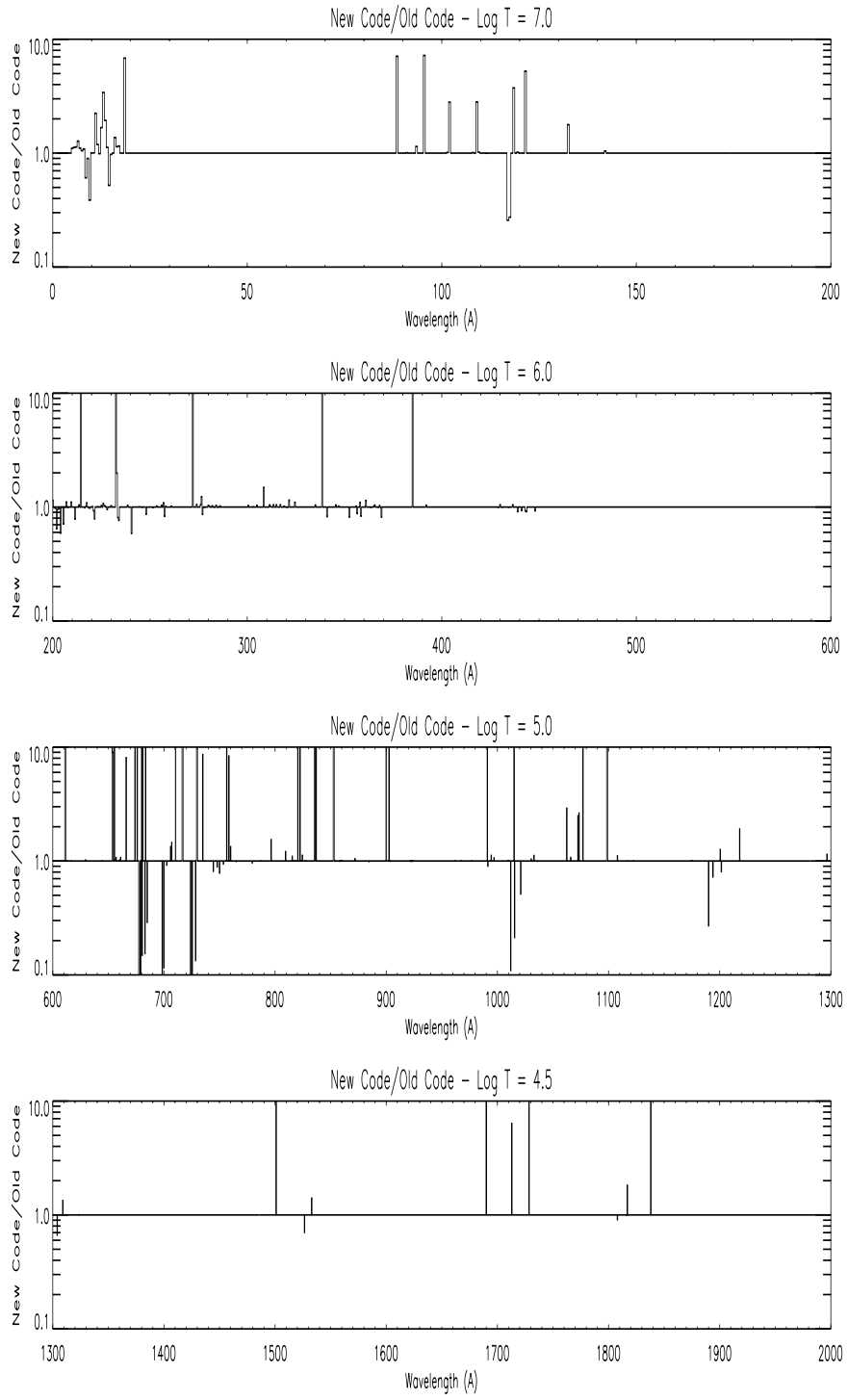

Fig. 13. Ratio between the emissivity of the new and of the old version of the Arcetri Code, calculated with $\log N_{\mathrm{e}}=10$ $\left(N_{\mathrm{e}}\right.$ in $\left.\mathrm{cm}^{-3}\right)$ and at different temperatures for each spectral range. The emissivity has been integrated over spectral bins each $0.5 \AA$ wide.

their lines show in Fig. 13 are due to changes in the wavelengths for several transitions, that have resulted from the revision of the wavelengths for these ions in CHIANTI version 3. Much more limited changes occur to the Arcetri Code between 280 and $650 \AA$.

The differences found between $650 \AA$ and $1400 \AA$ are due to the new ions inserted in the CHIANTI database in versions 2 and 3, to the increased number of energy levels in the atomic model of several ions, and to the wavelength revision done in version 3 . All these new data give rise to the large amount of spectral lines that cause the discrepancies in Fig. 13.

\section{Conclusions}

In the present work the revised version of the Arcetri Spectral Code is described. This new version includes all data from the CHIANTI version 3.03, and represent a significant improvement over the previous release of the Code in 1998. The main changes to the Code over the previous version are:

- the insertion of a large amount of data for the He-like and H-like sequences;

- the renewal of data for a large number of ions;

- a new assessment of wavelengths for a number of isoelectronic sequences.

Continuum radiation has been left unchanged.

The Code is made available through the internet at the address

\section{http://www.arcetri.astro.it/science/spettro/ spettro.html}

as a series of emissivity tables calculated as a function of electron temperature and density. Contribution Function for single emission lines, as well as total (line+continuum) emissivities integrated in $0.5 \AA$ steps are provided. These emissivities can be used for studies of both broad-band and high-resolution spectra emitted by optically thin astrophysical sources. Radiative losses for the most important elements are also provided.

A comparison has been performed between the present version of the Code and the earlier one by Landi \& Landini (1998), and the MEKAL Code (Mewe et al. 1995). The comparison with the earlier version of the Code was made to assess the effect of the introduction of the new CHIANTI 3.03 data into the Code database; the comparison with the MEKAL code was made to assess the completeness of the CHIANTI code against a completely independent database.

Results show that the CHIANTI 3.03 data introduce a large number of new transitions into the computed spectrum at all wavelengths, and remove a few other transitions which were overestimated or misplaced in the old version. Many of these transitions have been observed by space intrumentation.

The comparison with the MEKAL code shows that in the X-ray range the MEKAL code seems to include a greater number of lines, as witnessed by the stronger emissivity in the $13-16 \AA$ wavelength range. This is probably due to several transitions emitted by levels with principal quantum number $n=3,4,5$ in several highly ionized iron ions, not yet available in CHIANTI. At higher wavelengths the situation is reversed, as the Arcetri Code is able to predict many more lines than MEKAL. The latter is able to account for most of the strongest lines, but misses most of the weaker transitions, especially at the longest wavelengths.

Acknowledgements. The work of E. Landi has been supported by a grant from NASA's Applied Informations System 
Research Program (AISRP) and by the Office of Naval Research. M. Landini acknowledges support from the Italian Space Agency (ASI) and from the Italian Ministry for University and Research (MIUR). We wish to thank the referee, Dr. J. C. Raymond, for his very helpful comments.

\section{References}

Allen, C. W. 1973, Astrophysical quantities (The Athlone press, London)

Arnaud, M., \& Raymond, J. C. 1992, ApJ, 398, 394

Arnaud, M., \& Rothenflug, R. 1985, A\&AS, 60, 425

Bar-Shalom, A., Klapisch, M., \& Oreg, J. 2001, J. Quant. Spectrosc. Radiat. Transf., 71, 169

Bautista, M. A., \& Kallman, T. R. 2001, ApJS, 134, 139

Bhatia, A. K., Thomas, R. J., \& Landi, E. 2001a, ADNDT, in press

Bhatia, A. K., Landi, E., \& Mason, H. E. 2001b, ADNDT, in press

Brooks, D. H., Fischbacher, G., Fludra, A., et al. 2000, A\&A, 357,697

Butler, K., \& Zeippen, C. J. 1994, A\&AS, 108, 1

Daw, A., Parkinson, W. H., Smith, P. L., \& Calamai, A. G. 2000, ApJ, 533, 179

Del Zanna, G. 1999, Ph.D. Thesis, Univ. of Central Lancashire, UK

Dere, K. P., Landi, E., Mason, H. E., Monsignori Fossi, B. C., \& Young, P. R. 1997, A\&ASS, 125, 149

Dere, K. P., Young, P. R., \& Del Zanna, G. 2001, ApJS, 134, 331

Drake, J. J., \& Kashyap, V. 2000, Bull. Astr. Soc. India, 28, 475

Dupree, A. K. 1972, ApJ, 178, 527

Eissner, W., \& Seaton, M. J. 1972, J. Phys. B, 5, 2187

Eissner, W., Jones, M., \& Nussbaumer, H. 1974, Comp. Phys. Comm., 8, 270

Feldman, U. 1992, Phys. Scr., 46, 202

Feldman, U., \& Laming, J. M. 2000, Phys. Scr., 61, 222
Fuhr, J. R., Kelleher, D. E., Martin, W. C., et al. 1997, NIST Atomic Spectra Database ver. 2.0, NIST Physical Reference Data

Galavis, M., Mendoza, C., \& Zeippen, C. J. 1997, A\&AS, 123, 159

Grevesse, N., \& Anders, E. 1992, in Solar Interior and Atmosphere, ed. A. N. Cox, W. C. Livingston, \& M. S. Matthews (Tucson: Univ. Arizona Press), 1227

Grevesse, N., \& Sauval, A. J. 1998, Sp. Sc. Rev., 85, 161

Harrison, R. A., Sawyer, E. C., Carter, M. K., et al. 1995, Sol. Phys., 162, 233

Landi, E., \& Landini, M. 1998, A\&AS, 133, 411

Landi, E., Landini, M., Dere, K. P., Young, P. R., \& Mason, H. E. 1999, A\&AS, 135, 339

Landi, E., Feldman, U., \& Dere, K. P. 2002, ApJS, in press

Landini, M., \& Monsignori Fossi, B. C. 1990, A\&AS, 82, 229

Landini, M., \& Monsignori Fossi, B. C. 1991, A\&AS, 91, 183

Mazzotta, P., Mazzitelli, G., Colafrancesco, S., \& Vittorio, N. 1998, A\&AS, 133, 403

Mewe, R., Kaastra, J. S., \& Liedahl, D. A. 1995, Legacy, 6, 16

Meyer, J. P. 1985, ApJS, 57, 173

Monsignori Fossi, B. C., \& Landini, M. 1994a, The X-ray EUV spectrum of optically thin plasmas in The Sun as a variable Star, IAU Colloq. 143

Monsignori Fossi, B. C., \& Landini, M. 1994b, Sol. Phys., 152, 81

Monsignori Fossi, B. C., \& Landini, M. 1994c, ADNDT, 57, 125

Porquet, D., Mewe, R., Dubau, J., Raassen, A. J. J., \& Kaastra, J. S. 2001, A\&A, 376, 1113

Reisenfeld, D. B., Raymond, J. C., Young, A. R., \& Kohl, J. L. 1992, ApJ, 389, L37

Smith, R. K., Brickhouse, N. S., Liedahl, D. A., \& Raymond, J. C. 2001, ApJ, 556, 91

Shirai, J., Musgrove, A., \& Wiese, W. L. 2000, J. Phys. Chem. Ref. Data, Monograph 8

Shull, J. M., \& Van Steenberg, M. 1982, ApJSS, 48, 95

Waljeski, K., Moses, D., Dere, K. P., et al. 1994, ApJ, 429, 909 